\title{
Suitable Two Wheeled Vehicle Dynamics Synthesis for Interactive Motorcycle Simulator
}

\author{
Salim Hima* Lamri Nehaoua ${ }^{* *}$ Nicolas Séguy* \\ Hichem Arioui * \\ * IBISC, Informatique, Biologie Intégrative et Systèmes Complexes \\ FRE-CNRS 2873. e-mail : \{Salim.Hima, Nicolas.Seguy, \\ Hichem.Arioui\}@ibisc.univ-evry.fr. \\ ** INRETS, The French national institute for transport and safety \\ research.e-mail:nehaoua@inrets.fr
}

\begin{abstract}
This paper describes a modelling technique for deriving the motorcycles equation of motion. Based on the recursive Newton-Euler approach adapted to tree structure with floating base multibody systems, the derived model presents a low number of arithmetic operations, and hence, is suitable for implementation into a two wheeled vehicles simulator and other model based real-time application. The synthesized model takes in consideration the main forces and moments affecting the behavior of the motorcycle such as: pneumatic, aerodynamic, suspensions, contact constraints and control inputs.
\end{abstract}

Keywords: Motorcycle driving simulator, motorcycle dynamics, Recursive Newton-Euler algorithm.

\section{INTRODUCTION}

Motion simulators have known a wide utilization in different area of vehicle purposes. They were initially developed in aeronautic field as a tool to training a novice pilots and also, to evaluate the performances of a new design in a safe environment. The success known in this field, have motivated an extension of this technology to other types of vehicles especially cars and recently motorcycles. It is possible to distinguish two main categories of simulators: fixed and motion base simulators. The former can merely provide the rider with visual and auditive cues, while the second add an appropriate inertial cue component to render the perception more realistic and ensure a good immersion of the rider in a virtual traffic environment. Feeding the rider by an acceptable inertial cue is possible by adequately stimulate his vestibular organ with motions generated by moving the simulator motion-base, on which he is rising, within its limited workspace. In literature, the Gough-Stewart mechanism is widely used as motion base platform, especially in heavy simulators. In the framework of this project, a special low cost motion-base platform adapted to motorcycle purposes has been proposed, see Nehaoua (2007). This simulator is conceived to reproduce the most significant situations in real riding, needed for training new riders and also to enhance performances of experienced ones in risky situations.

Like all simulators, a motorcycle behavior component (forward dynamics) is essential to supply the different simulator modules with information needed for their functionality, in response to rider actions. In literature, pre-

\footnotetext{
ऋ This work was supported by the French National Research Agency (ANR) in the Framework of SIMACOM project.
}

sented models are mostly developed for control objectives and focused on local behaviors. However, in the scope of simulators, a high fidelity inertial cues reproduction needs an accurate motion model which cover a wide range of motorcycle behavior and fulfill real-time constraints.

Considering the motorcycle as a set of rigid bodies connected between them by simple joints, multibody system mechanics theory offers a convenient framework to derive its motion model. In Cossalter (2002), authors have adopted Lagrange formalism to derive their motorcycle motion model. A direct application of this approach, leads to unattractive performances in term of number of operations and implementation facilities. An algorithmic formulation alternative of this formalism, that presents the advantage to be easily debugged and implemented, has been presented in Hima (2007).

Exploiting the recursive scheme in computing some kinematic quantities, very efficient algorithms have been proposed to solve the inverse dynamics of open chain multibody systems, see Featherstone (2000); Khalil (1987); Hollerbach (1980). So, the direct dynamics can be derived from the inverse dynamics using the articulated body algorithm (ABA), Featherstone (2000). This algorithm is known to be fast, accurate and stable, see Khalil (1999). In this paper, we extend $\mathrm{ABA}$ algorithm to derive a fast direct model of motorcycles suitable for two wheeled interactive simulator. In the second section of this paper, a presentation of our simulator is given. Third Section is devoted to the derivation steps of motorcycle forward dynamics. In the fourth section, simulation results are illustrated. Finally, conclusion remarks and future works are outlined. 


\section{SIMACOM PROJECT PRESENTATION}

The primary goal of the SIMACOM project is to propose a low cost replicable motorcycle simulator. It's dedicated to driving schools for training novice riders, performing the experienced riders aptitudes face to life-threatening situations and study the rider-motorcycle interaction in a safe environment. Mainly, the proposed simulator architecture is designed around five subsystems: a visual subsystem, an acoustic subsystem, a motion-base platform, motorcycle model and haptic subsystems, see Fig 1 . The visual subsystem immerse the rider in a virtual traffic environment, managed by the ARCHISIM software, and provides him with velocity cue.

In Panerai (2001) the authors have shown that, an acoustic cues deprivation leads to a systematic increase tendency of the speed by drivers. To prevent this problem, acoustic subsystem is placed to reproduce sounds resulting from the juxtaposition of many sources where the main ones come from the engine and tires. In addition, inertial cues are generated by stimulating the rider vestibular organ by moving him with proper motions. For this aim, a motionbase platform, which consists of a real motorcycle structure hanged on a parallel robot, is designed to reproduce the main motorcycle situations such as bending in braking situation, leaning in cornering situation and also the rear tire skidding situation. That's why, two prismatic actuator are placed in the front and connected to saddle body by way of spherical joints. So, the pitch and roll of the motion-base are directly controlled by changing the legs lengths using two DC motors. In addition, a sliding system is placed at the rear of the motion-base which allows a horizontal displacement to create illusion for rear tire skidding situation, see Fig 2.

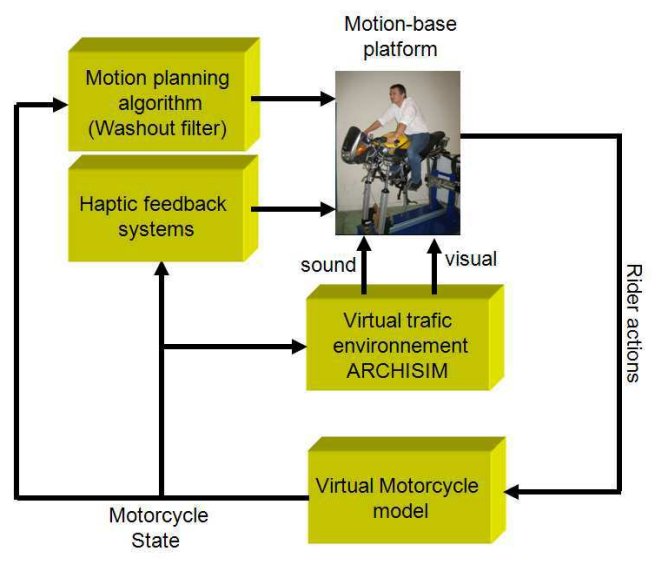

Fig. 1. SIMACOM project simulator architecture

Changes in previous described subsystems states are made in response to the rider actions captured by placing sensors in the motorcycle control organs (throttle angle, braking and clutch leavers). Ones rider's action are sensed, they are sent to the motorcycle dynamics model in order to compute motorcycle's motion (configuration, velocities and accelerations). These informations are then transmitted to visual and acoustic subsystem to update the virtual environment and to generate adequate sounds. In the same time, motion informations are also provided to a washout filter in order to transform it into motions compatible with motion-base workspace limitations.

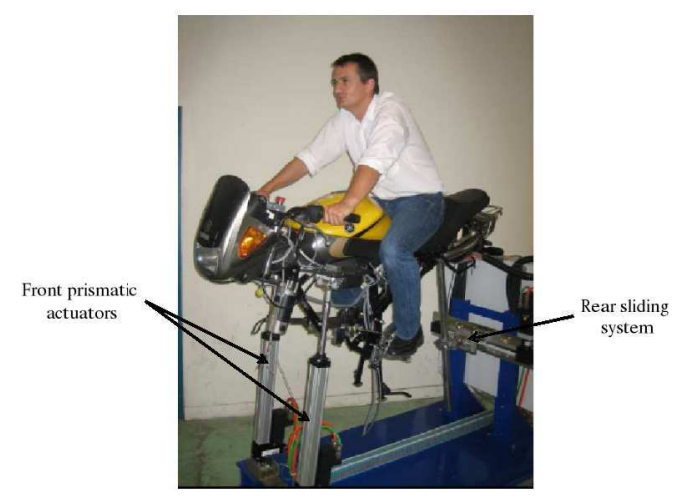

Fig. 2. Motion-base platform prototype

When riding a motorcycle, the ground-tire interaction forces and the gyroscopic precession force of the front wheel are transmitted to the handlebar and so felt by the rider. Furthermore, tension forces exerted on the rider arms in acceleration and deceleration phases, caused by the rider bust inclination, are present. In order to reproduce these forces and make the simulator more efficient, two haptic feedback subsystems are integrated in our simulator (Fig 3). The first subsystem consists of a DC motor connected to the steering axis by way of pulley-belt system. It permits the application of a torque on the steering axis to give the rider sensation of the tire-ground and gyroscopic forces. This torque is computed from the motorcycle dynamics model. Moreover, the second subsystem consists of a mechanism that endow the handlebar with a longitudinal translation motion controlled by a brushless actuator, see Fig 3. In fact, in acceleration phase, the handlebar translates far from the rider and hence, pulls the rider arms to create illusion of the bust inclination away from the handlebar. Otherwise, in deceleration phase, the handlebar translates toward the rider and so, pushes the rider arms to create illusion of the bust inclination toward handlebar.

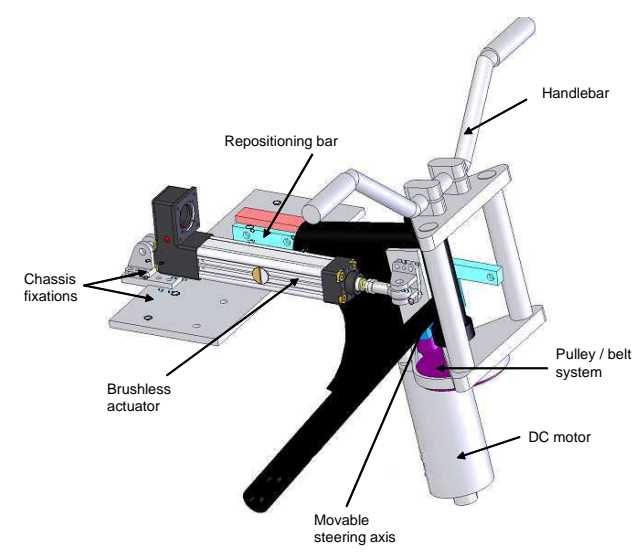

Fig. 3. Haptic feedback systems in the handlebar 
For further description of this simulator and kinematic study of the motion-base, readers can consult Nehaoua (2007).

\section{MOTORCYCLE MODELLING}

Motorcycle direct dynamics is the study of its behavior under rider actions. This relationship can be derived from mechanics theory. Indeed, motorcycle is a system composed by a set of rigid bodies connected with joints. We have considered six bodies: the saddle body, the front upper body (handlebar and upper part of suspension), the front lower part (lower part of suspension), the swinging arm and the two tires. The handlebar and the swinging arm are attached to the saddle body by a simple rotoid joints. The font lower part is linked to the front upper part by a prismatic joint. In addition, the rear suspension is connected to the saddle with a rotoid joint from one side and to the swinging arm with a rotoid joint also on the other side. This creates a closed kinematic loop, and so, leads to modelling difficulties. Finally, rear and front wheels are respectively connected to the other tips of swinging arm and front lower part as sketched in Fig 4.

The motion of the motorcycle is referenced to an inertial frame $\mathcal{R}_{0}\left(O_{0}, x_{0}, y_{0}, z_{0}\right)$. To each body $B_{i}$ an orthonormed frame was rigidly attached. In order to completely define the configuration of the motorcycle, a set of 11 DOFs has been considered. A full characterization of motorcycle's situation can be done by the position and orientation of the saddle $(x, y, z, \phi, \theta, \psi)$, the steering angle or handlebar , $\delta$, the elongation of the front suspension, $L$, the rotation angle of the swinging arm with respect to the saddle, $\theta_{s}$ (image of rear suspension elongation), and finally, the rotation angles of the front and rear tires, $\theta_{f}$ and $\theta_{r}$.

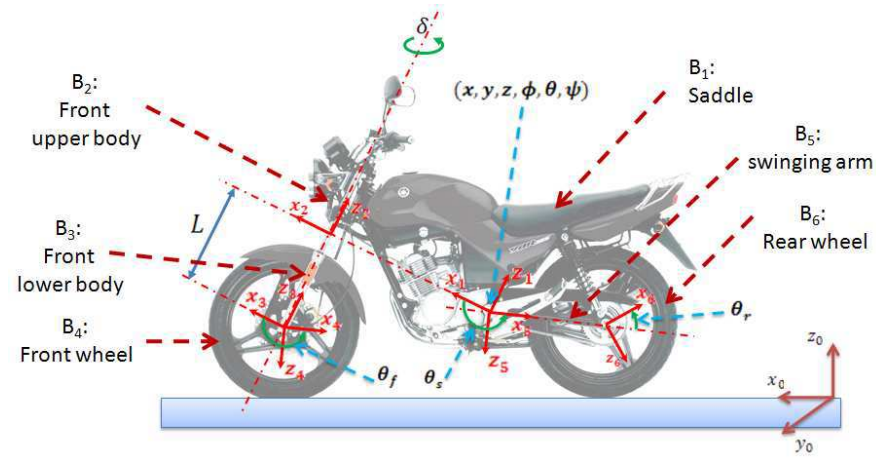

Fig. 4. Motorcycle configuration parametrization

\subsection{Direct Dynamics}

A direct application of the classical formalisms from mechanics of multiboby systems can lead to an inefficient dynamic model in term of the number of arithmetic operations. However, recursive techniques present another alternative to greatly reduce the computational burden and that should allow a real-time computation of the direct dynamics of multibody systems. In Khalil (1999) and Hollerbach (1980), Recursive Newton-Euler Algorithm, RENA, algorithm have been shown to be a fast one. Originally, this technique is developed to solve the inverse dynamic model of the open chain manipulator with a fixed base for control purposes. By projecting the dynamics of each body in its attached frame, the acceleration of the joint variable can be easily derived without need to compute the total inertia matrix of the whole system and invert it. In addition, this technique, named Articulated Body Algorithm (ABA), is more numerically stable than the inertia matrix inversion method, see Khalil (1999).

To illustrate the ABA, consider the multibody system sketched in Fig 5. Let $\mathbb{V}_{i}=\left(v_{i}^{T}, \omega_{i}^{T}\right)^{T}$ be the twist vector of $B_{i}$ where $v_{i}$ is its linear velocity and $\omega_{i}$ is its angular velocity. $\mathbf{k}_{i}$ is the $i^{t h}$ joint axis, $\mathbb{F}_{i}^{e}=\left(F_{i}^{e T}, M_{i}^{e T}\right)^{T}$ is the external wrench acting on $B_{i}, \mathbf{L}_{i}$ the position vector of $(i+1)^{t h}$ joint origin with respect to $i^{t h}$ joint origin, $\mathbf{S}_{i}$ is the position vector of the $B_{i}$ center of gravity with respect to the $i^{\text {th }}$ joint origin, ${ }^{i} \mathbf{R}_{i-1}$ the transformation matrix from $B_{i-1}$ to $B_{i}, \mathbb{F}_{i}=\left(F_{i}^{T}, M_{i}^{T}\right)^{T}$ is the link wrench vector transmitted to $B_{i}$ from $B_{i-1}, m_{i}$ and $\mathbf{J}_{i}$ are, respectively, the mass and the inertia tensor of the body $B_{i}, q_{i}$ is $i^{t h}$ joint variable and $\tau_{i}$ be the actuator force/torque acting on the joint $i$. In the sequel of this paper, the expression ${ }^{i} \mathbf{X}_{j}$ means that the vector $\mathbf{X}_{j}$ is projected into the frame associated to $B_{i}$.

Let $\sigma_{i}$ be a binary variable defining the joint type and given by:

$$
\sigma_{i}= \begin{cases}1 & \text { if joint } \mathrm{i} \text { is prismatic } \\ 0 & \text { if joint } \mathrm{i} \text { is revolute }\end{cases}
$$

Let ${ }^{i} \mathbb{T}_{i-1}$ be a $6 \times 6$ matrix defined as:

$$
{ }^{i} \mathbb{T}_{i-1}=\left(\begin{array}{cc}
{ }^{i} \mathbf{R}_{i-1} & -{ }^{i} \mathbf{R}_{i-1}{ }^{i-1} \tilde{\mathbf{L}}_{i-1} \\
0_{3 \times 3} & { }^{i} \mathbf{R}_{i-1}
\end{array}\right)
$$

where $\sim$ is the skew-symmetric matrix operator for a vector $\mathbf{v}=(x, y, z)^{T}$ defined by:

$$
\tilde{\mathbf{v}}=\mathbf{v} \times=\left(\begin{array}{ccc}
0 & -z & y \\
z & 0 & -x \\
-y & x & 0
\end{array}\right)
$$

Let ${ }^{i} \mathbb{J}_{i}$ be the global inertia matrix of $B_{i}$ given by:

$$
{ }^{i} \mathbb{J}_{i}=\left(\begin{array}{cc}
m_{i} \mathbf{I}_{3 \times 3} & -m_{i}{ }^{i} \tilde{\mathbf{S}}_{i} \\
m_{i}{ }^{i} \tilde{\mathbf{S}}_{i} & \mathbf{J}_{i}
\end{array}\right)
$$

We define ${ }^{i} \mathbf{a}_{i}=\left[\sigma_{i}{ }^{i} k_{i}^{T}, \bar{\sigma}_{i}{ }^{i} k_{i}^{T}\right]^{T}$.

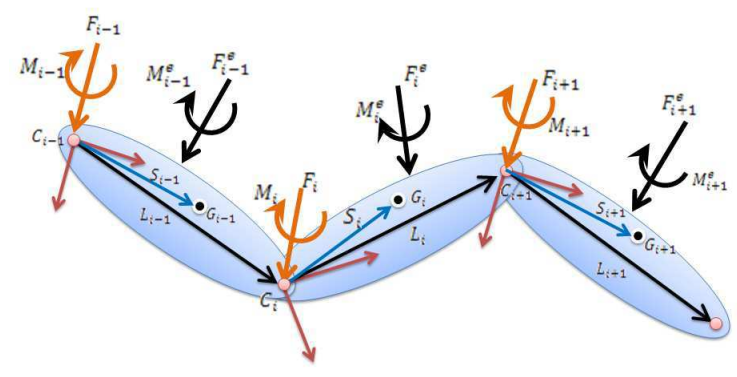

Fig. 5. Open chain multibody system

The ABA operates by three recursions. In the first forward recursion, velocities, coriolis, centrifugal and external forces are computed for each body. The second backward 
recursion, calculates the total forces and inertia matrices felt by each joint. Finally, the third recursion computes the acceleration of each joint and dynamic torsor applied to each joint. Setting $\omega_{0}=\dot{\omega}_{0}=0$ and $\mathbb{V}_{0}=\left(-\mathbf{g}, 0_{1 \times 3}\right)^{T}$, where $\mathbf{g}$ is the gravity vector, the ABA is given by:

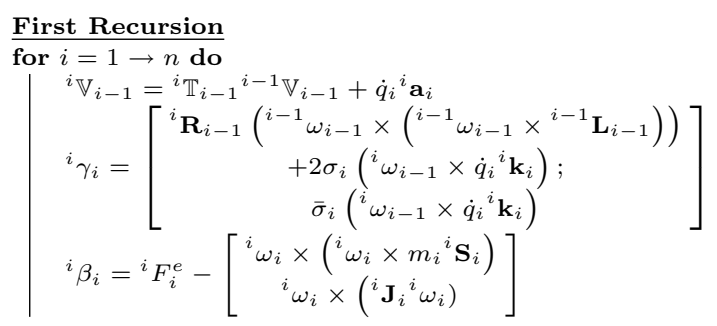

end

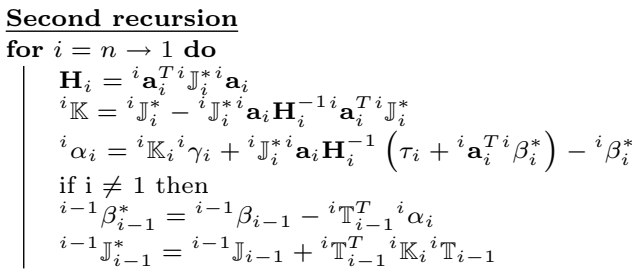

end

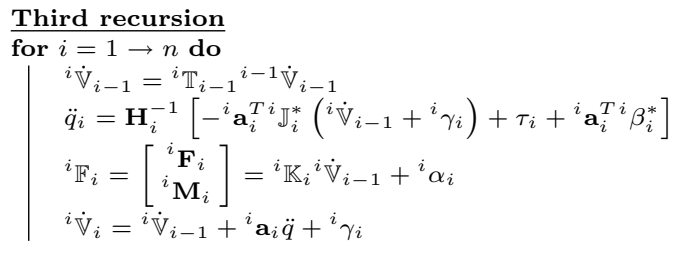

end

Algorithm 1. Recursive Newton-Euler algorithm for direct dynamics

Removing the rear suspension and replacing it with two forces acting on the saddle and the swinging arm at rear suspension fixation points, the motorcycle can be considered as a tree structure system with floating base. Hence, algorithm 1 is used to compute the accelerations of relative configurations $\left(\delta, L, \theta_{f}, \theta_{s}, \theta_{r}\right)$, and forces acting on their corresponding articulations for the open chains $\left(B_{2}, B_{3}, B_{4}\right)$ and $\left(B_{5}, B_{6}\right)$. At this stage, the remainder configuration accelerations can be computed by considering the saddle body only, and replacing the front and the rear chains by transmitted dynamical wrenches $\left(\mathbb{F}_{2}, \mathbb{F}_{5}\right)$ at the $2^{\text {nd }}$ and $5^{\text {th }}$ joints origins. The direct model of the saddle is then given by:

$$
\begin{aligned}
{ }^{1} \dot{\mathbb{V}}_{1} & ={ }^{1} \mathbb{J}_{1}^{-1}\left(\mathbf{C}\left(\eta,{ }^{1} \mathbb{V}_{1}\right)+\mathbf{G}(\eta)+{ }^{1} \mathbb{F}_{2}+{ }^{1} \mathbb{F}_{5}+{ }^{1} \mathbb{F}_{1}^{e}\right) \\
\dot{\eta} & =\mathbb{R}^{1} \mathbb{V}_{1}
\end{aligned}
$$

where $\eta=(x, y, z, \phi, \theta, \psi)$ is the configuration vector of the saddle. ${ }^{1} \mathbb{V}_{1}$ is the vector of the linear and angular velocities of saddle body with respect to its frame. ${ }^{1} \mathbb{J}_{1}$ is the inertia matrix of the saddle body expressed in its frame. $\mathbf{C}$ and $\mathbf{G}$ are, respectively, the Centrifugal/Coriolis and weight wrenches. ${ }^{1} \mathbb{F}_{1}^{e}$ is the external wrench acting on the saddle. $\mathbb{R}$ is the transformation matrix from $B_{1}$ frame to the inertial frame $\mathcal{R}_{0}$ and can be found in Shabana (1989).

\subsection{External forces}

In this section, we summarize the principal forces and moments affecting the motorcycle behavior.

Suspension forces In order to absorb the shocks caused by the road irregularities and bumps, the motorcycle is equipped with rear and front suspension systems. In this paper, a linearly dependence of the forces generated by the suspensions on the elongations and their rates of change is considered:

$$
F_{i}=K_{i}\left(L_{i}-L_{i 0}\right)+C_{i} \dot{L}_{i}
$$

where $i=\{r s, f s\}$ designates the rear or front suspension, $L_{i 0}$ is its free load elongation, $K_{i}$ is the suspension stiffness and $C_{i}$ is the damper coefficient. According to Fig 6, the suspensions lengths can be expressed as:

$$
L_{i}=\sqrt{\left({ }^{j} P_{i_{u}}-{ }^{j} P_{i_{l}}\right)^{T}\left({ }^{j} P_{i_{u}}-{ }^{j} P_{i_{l}}\right)}
$$

where $P_{i_{u}}$ and $P_{i_{l}}$ denote respectively the position of the upper and lower extremities of suspension $i .(i, j) \in$ $\{(f s, 2),(r s, 1)\}$. Hence, the rate of change of suspensions lengths are given by:

$$
\dot{L}_{i}=\frac{1}{L_{i}}\left({ }^{j} \dot{P}_{i_{u}}-{ }^{j} \dot{P}_{i_{l}}\right)^{T}\left({ }^{j} P_{i_{u}}-{ }^{j} P_{i_{l}}\right)
$$

Hence, the suspensions wrenches are given by:

$$
\begin{aligned}
{ }^{1} \mathbb{F}_{r s_{u}}=\left[\frac{\left({ }^{1} P_{r s_{u}}-{ }^{1} P_{r s_{l}}\right)^{T}}{L_{r s}} F_{r s},{ }^{1} P_{r s_{u}} \times \frac{\left({ }^{1} P_{r s_{u}}-{ }^{1} P_{r s_{l}}\right)^{T}}{L_{r s}} F_{r s}\right]^{T} \\
{ }^{5} \mathbb{F}_{r s_{l}}=\left[\frac{\left({ }^{5} P_{r s_{l}}-{ }^{5} P_{r s_{u}}\right)^{T}}{L_{r s}} F_{r s},{ }^{5} P_{r s_{l}} \times \frac{\left({ }^{5} P_{r s_{l}}-{ }^{5} P_{r s_{u}}\right)^{T}}{L_{r s}} F_{r s}\right]^{T} \\
{ }^{2} \mathbb{F}_{f s_{u}}=\left[\frac{\left({ }^{2} P_{f s_{u}}-{ }^{2} P_{f s_{l}}\right)^{T}}{L_{f s}} F_{f s},{ }^{2} P_{f s_{u}} \times \frac{\left({ }^{2} P_{f s_{u}}-{ }^{2} P_{f s_{l}}\right)^{T}}{L_{f} s} F_{f s}\right]^{T} \\
{ }^{3} \mathbb{F}_{f s_{l}}=\left[\frac{\left({ }^{3} P_{f s_{l}}-{ }^{3} P_{f s_{u}}\right)^{T}}{L_{f s}} F_{f s},{ }^{3} P_{r s_{l}} \times \frac{\left({ }^{3} P_{f s_{l}}-{ }^{3} P_{f s_{u}}\right)^{T}}{L_{f s}} F_{f s}\right]^{T}
\end{aligned}
$$

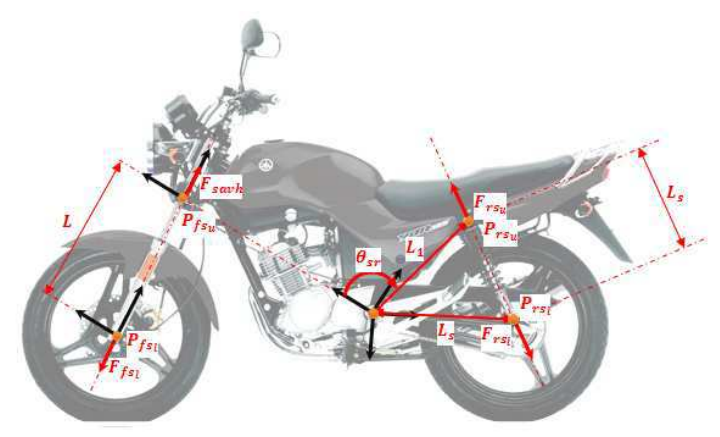

Fig. 6. Suspension systems wrenches

Tire/road contact wrench Behavior of ground vehicles depends significantly on the nature of the interaction between the tire and the road. Indeed, in the last two 
decades, and due to the importance of this phenomena in the security of ground vehicles, several efforts are done to establish mathematical models characterizing the friction forces and moments of the tire/road contact. The well known and widely used one is Pacejka Magic formula. This model captures in steady-state motion, the tire/road forces and moments, in algebraic equations form, with respect to kinematic quantities (longitudinal slip $\kappa$, lateral slip $\beta$, the load $F_{z}$ ). The steady-state behavior restriction still valid in the scope that, the effect of the tire dynamics is generally small compared to the effect of the complete motorcycle dynamics.

Initially, Pacejka model has been developed for four wheels vehicles. In this case, wheels stay approximately vertical and hence, the camber angle can be neglected. Other varieties of this model have been proposed and adapted to motorcycle tires by including the camber angle. Indeed, this angle could achieves $50^{\circ}$ for racing motorcycles when cornering and hence, could'nt be ignored, Lot (2004).

Among a six components of the tire/road contact wrench, we have considered the principal ones: longitudinal and lateral forces and the aligning moment, Sharp (2004); Pacejka (1997). Fig 7 illustrates the shape of longitudinal force, lateral force and aligning moment vs longitudinal slip, for different values of lateral slip angle, corresponding to experimental identified data of the $160 / 70$ tire type found in Sharp (2004).
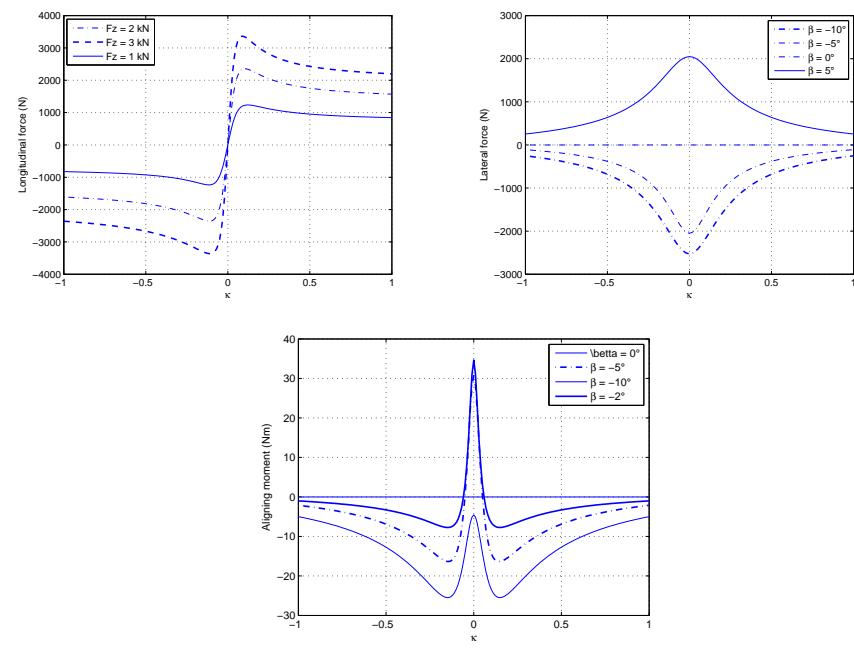

Fig. 7. Longitudinal and lateral forces and aligning moment for 160/70 motorcycle tire experimental model from Sharp (2004)

Aerodynamic wrench When the motorcycle moves, its interaction with the air generates an aerodynamic forces and moments. The most important components are the drag and the lift applied at the center of pressure, in general, located above the center of gravity of the motorcycle. The consequence of this deportation is the presence of an aerodynamic pitching moment. The drag force influences the maximum achievable speed and also acceleration performance. The lift force can have an undesirable effects on the motorcycle performances. Indeed, it reduces the load on the wheels and, thus, decreases the tire adherence. Mainly, the drag and the lift forces are proportional to the square of the motorcycle linear velocity relative to air:

$$
F_{D}=\frac{1}{2} C_{D} \rho S V_{a}^{2} ; \quad F_{L}=\frac{1}{2} C_{L} \rho S V_{a}^{2}
$$

where $C_{D}$ and $C_{l}$ are dimensionless coefficient, $\rho$ is the air density, $V_{a}$ is the motorcycle relative velocity and $S$ is the frontal motorcycle area exposed to the air flow.

Contact constraints forces Keeping the tires in contact with the road imposes two unilateral constraints. In order to satisfy these constraints, we have placed two springdamper systems between the road and the tires contact points. The forces of the spring-damper systems act solely when the tires penetrate into the ground. This formulation can also capture tires carcasses elasticity features, see Fig 8.

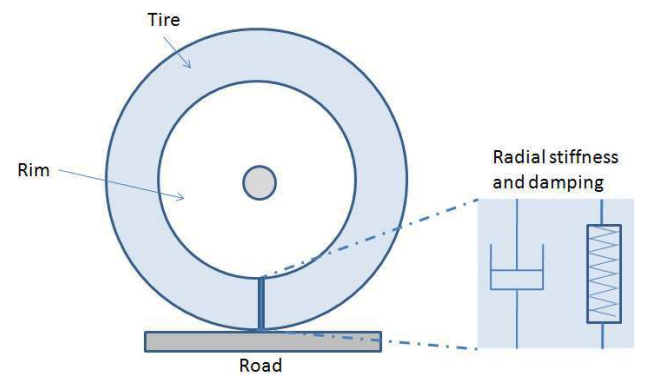

Fig. 8. Radial elasticity of the tire carcass

This formulation is more advantageous than using Lagrangian multipliers which need to include constraints stabilisers to prevent constraints divergence.

Input controls The last considered external forces acting on the motorcycle behavior are coming from the rider actions through the engine torque, brakes torques and finally steering torque. We have modeled these actions by a pure torque and we don't integrate their dynamic models, which will be considered in future works.

\section{SIMULATION RESULTS}

To test the behavior of the developed dynamic model of the motorcycle, we have synthesized an autopilot based on a linear PID controller for longitudinal speed regulation. This controller acts directly on the rear tire velocity by applying an acceleration/braking torque. The control torque is then filtered by a first order filter to emulate the delay of the engine reaction, and bounded by the maximum torque admissible by the engine at the rear tire axis. For this simulation, the desired velocity profile consists of three main phases, acceleration phase up to $30 \mathrm{~m} / \mathrm{s}$, deceleration phase down to $15 \mathrm{~m} / \mathrm{s}$, and finally an acceleration phase again up to $20 \mathrm{~m} / \mathrm{s}$, see Fig 9 . In this figure, the steady error between the desired and the motorcycle errors in the three phases are different. It is most important when the motorcycle travels with a high speed. The origin of this effect is related to the aerodynamic drag force which is proportional to the square of the motorcycle speed. In Fig 10, requested autopilot control torque and effective control torque delivered by the engine at the rear tire axis are sketched. The effect of the engine delay and torque limitation can be observed on the convergence time of the speed profile. Fig 11 illustrates the effective motorcycle 
driving forces generated by the rear and the front tires pneumatic models.

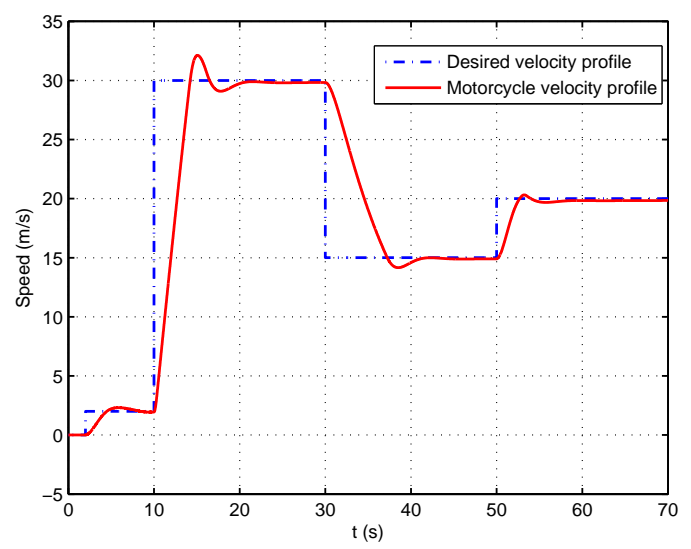

Fig. 9. Reference and real motorcycle speeds

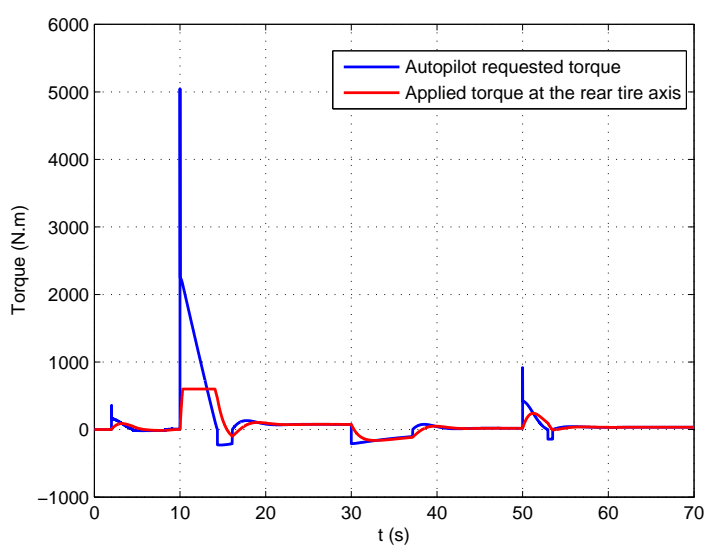

Fig. 10. Comparison of the requested control and engine delivered control
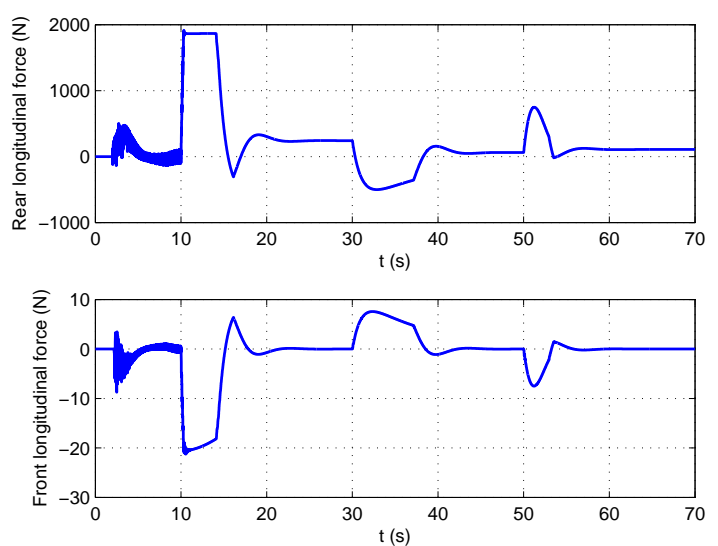

Fig. 11. Rear and front longitudinal tires forces

\section{CONCLUSION AND FUTURE WORKS}

Based on Recursive Newton-Euler algorithm, a motorcycle dynamic model dedicated to the driving simulator application is proposed. The development method is chosen to reduce the calculation burden, and hence, reduce the delay time between the application of the rider actions instant and the change made in the motorcycle dynamics in response to these actions.

As future works, a more complete model will be proposed including a refined brakes models, an accurate engine dynamics model and the tire/road contact point migration in the tire circumference when cornering. Moreover, a quantitative evaluation will be done for real-time applications. Finally, the assisted rider systems will be conceived and tested on the simulator.

\section{REFERENCES}

L. Nehaoua, S. Hima, H. Arioui, N. Séguy and S. Espié Design and Modeling of a New Motorcycle Riding Simulator. American Control Conference, pages 176-181, 2007.

W. Khalil and J. F. Kleinfinger Minimum operations and minimum parameters of the dynamic models of tree structure robots. IEEE Journal on Robotics and Automation, Vol RA-3, NO. 6, 1987.

W. Khalil and E. Dombre. Modélisation, identification et commande des robots. Hermes science publications, Paris, 2nd edition, 1999.

J. M. Hollerbach A recursive lagrangian formulation of manipulator dynamics and a comparative study of dynamics formulation complexity. IEEE Transaction on systems, man and cybernetics, vol smc-10, No 11, pages 730-736, 1980.

F. Panerai et al Speed and safety distance control in truck driving: comparison of simulation and real-world environment. Proceedings of driving simulation conference, pages 91-107, 2001.

A. Champion, Ming-Yu Zhang, J. M. Auberlet and S. Espie, Behavioral simulation of a high-density traffic network involving an adaptive ramp metering system. IEEE International Conference on Systems, Man and Cybernetics, vol 5, 2002.

V. Cossalter and R. Lot, A Motorcycle Multi-Body Model for Real Time Simulations Based on the Natural Coordinates Approach. Vehicle System Dynamics, vol 37, No. 6, pages 423-447, 2002.

S. Hima, L. Nehaoua, N. Séguy and H. Arioui, Motorcycle Dynamic Model Synthesis for Two Wheeled Driving Simulator. International IEEE conference on intelligent transportation systems, 2007.

R. Featherstone and D. Orin, Robot dynamics: equations and algorithms. IEEE International Conference on Robotics and Automation, Vol. 1, pages 826-834, 2000.

A. A. Shabana Dynamics of multibody systems. John wiley \& sons, 1989 .

R. Lot, A Motorcycle Tire Model for Dynamic Simulations: Theoretical and Experimental Aspects. Meccanica, an International Journal of Theoretical and Applied Mechanics, Vol. 39, pages 207-220, 2004.

R. S. Sharp and S. Evangelou and D. J. Limebeer, $A d-$ vances in Modelling of Motorcycle Dynamics. Multibody System Dynamics, Vol. 12, pages 251-283, 2004.

H. B. Pacejka and I. J. M. Besselink, Magic Formulation Tyre Model with Transient Properties. Vehicle System Dynamics, Vol. supplement 27, pages 234-249, 1997. 